\title{
Increased risk of bacteremia in patients hemodialyzed through central catheters
}

\author{
Geoffrey Taylor, MD, Teresa Kirkland, BScN, Peter Hamilton, MBBCH
}

\begin{abstract}
As part of an ongoing prospective survey of nosocomial bacteremias, patients developing bacteremia while undergoing in-centre hemodialysis were observed over a 23 month period. Thirty-six episodes of bacteremia occurred in 30 patients; every episode was directly attributable to hemodialysis. In 28 of the 36 episodes $(78 \%)$, there was evidence of inflammation with or without drainage of pus at the hemodialysis access site. Staphylococcus aureus accounted for $76 \%$ of the
\end{abstract}

bacteremic isolates. Patients hemodialyzing through central venous catheters had a far higher incidence of bacteremia ( 0.01 per dialysis run) than patients hemodialyzing through vascular grafts $(0.0005$ per dialysis run). Can J Infect Dis 1990;1(1):11-14

Key Words: Bacteremia, Hemodialysis, Staphylococcus aureus
TNEECTION IS NOW THE PRINCIPAL CAUSE OF MORbidity and the second leading cause of death in patients undergoing hemodialysis, with vascular access the principal source of these infections $(1,2)$. As part of a hospital-wide program of monitoring blood culture isolates for nosocomial bacteremias, the authors have been prospectively following patients acquiring bacteremia as a result of in-centre hemodialysis. The results of two years of such surveillance are presented, and evidence provided that hemodialysis through a percutaneous central venous access catheter greatly adds to the risk of dialysis-related bacteremia.

University of Alberta Hospitals, Edmonton. Alberta Correspondence and reprints: Dr GD Taylor, 2E3.11 WMC, University of Alberta Hospitals. Edmonton. Alberta T6G 2B7 Received for publication January 25, 1990. Accepted April 3, 1990

\section{PATIENTS AND METHODS}

As part of an ongoing infection control surveillance project identifying nosocomial bacteremia, hemodialysis-related bacteremic episodes occurring within the in-centre hemodialysis unit were prospectively evaluated over a 23 month period from August 1, 1986 to June 30, 1988. One of the authors (TK) reviewed all positive blood cultures reported by the hospital microbiology laboratory. Bacteremic cases were reviewed by a physician (GT) prior to inclusion. All bacteremias occurring in patients undergoing hemodialysis in the 16bed, in-centre hemodialysis unit were documented. Patients undergoing dialysis for acute renal failure in the intensive care units were not included. A bacteremia was defined as a bacterial infection of the bloodstream where one or more blood cultures contained pathogenic organisms. If culture yielded a typical skin commensal, two 
blood cultures taken at different sites or at different times positive for the same species of bacteria were considered to represent bacteremia. One blood culture with a typical skin commensal was documented as a bacteremia if the attending physician diagnosed bacteremia and prescribed a course of antibiotic therapy directed against the bacteremic organism. Organisms considered to be skin commensals were: Corynebacterium species, Bacillus species, viridans group streptococci, micrococcus, coagulase-negative staphylococci or Propionibacterium species. A single positive culture due to Staphylococcus aureus was considered significant. The primary organ system causing the bacteremia, if present, was noted, and the mode of hemodialysis at the time of bacteremia was determined (ie, via a vascular access graft or through central venous cannula). Evidence of access site infection was determined by direct inspection. Infection rates were calculated per dialysis run and per dialysis year (one patient undergoing 156 hemodialysis runs was equivalent to one dialysis year).

The University of Alberta Hospitals (UAH) dialysis centre is a 16-bed unit providing in-centre hemodialysis services to an average of 100 patients, up to 283 runs/week. Patients are preferentially dialyzed through an arteriovenous fistula. Dialysis through a central venous catheter is undertaken if no fistula is in place or if the fistula is actively infected or clotted. The right subclavian site is preferentially used: insertion is performed in the unit by house staff using the Seldinger

\section{TABLE 1}

Causative organisms in 36 episodes of hemodialysis-related bacteremia

\begin{tabular}{lc}
\hline Organism & Episodes (\%) \\
\hline Staphylococcus aureus & $27(75 \%)$ \\
Klebsiella pneumoniae & $3(8 \%)$ \\
Enterococcus fecalis & $3(8 \%)$ \\
Candidaspecies & $1(3 \%)$ \\
Coagulase-negative staphylococcus & $1(3 \%)$ \\
Escherichia coli & $1(3 \%)$ \\
Total & $36(100 \%)$ \\
\hline
\end{tabular}

TABLE 2

Bacteremia by method of dialysis

\begin{tabular}{lccc}
\hline $\begin{array}{l}\text { Dialysis } \\
\text { method }\end{array}$ & $\begin{array}{c}\text { Episodes of } \\
\text { bacteremia }\end{array}$ & Dialysis runs & $\begin{array}{c}\text { Episodes of } \\
\text { bacteremia per } \\
\text { dialysis run }\end{array}$ \\
\hline $\begin{array}{l}\text { Central } \\
\text { venous line }\end{array}$ & 24 & 2399 & $0.01^{\circ}$ \\
$\begin{array}{l}\text { Vascular graft } \\
\text { Total }\end{array}$ & 12 & 23,089 & 0.0005 \\
\hline
\end{tabular}

- $P=0.001$ by $\chi^{2}$ test. Odds ratio $19.4 .95 \%$ confidence interval 9.3 to 41.2 technique, as described by Schwarzheek et al (3). Prophylactic antibiotics are not administered prior to line insertion, but may be given if a concurrent infection is present elsewhere, eg, fistula infection. The line is used exclusively for dialysis and is flushed with 2000 to 3000 units of heparin at the time of insertion and after each dialysis run. Transparent semiocclusive dressings are applied and changed after each run and when the site is inspected. Povidone-iodine ointment is applied to central line insertion sites at the time of dressing change. The catheter is removed when no longer required for hemodialysis or if there is catheter malfunction, thrombosis or evidence of local (pus or erythema) or systemic infection. Routine changing of the subclavian line, either by reinsertion or by passing over a guidewire to the same site, is not undertaken.

\section{RESULTS}

Thirty-six episodes of bacteremia were detected in 30 patients. No source of infection apart from the dialysis access site was found in any patient; in 28 episodes $(78 \%)$ there were clinical signs of infection (inflammation with or without drainage of pus) at the access site. Staph aureus was by far the most common isolate, accounting for 27 episodes $(75 \%)$ (Table 1$)$. There was one death in the bacteremia population which was clinically directly attributed to infection with Klebsiella pneumoniae (bacteremia mortality rate $2.7 \%$ ). Dialysis via a central venous catheter was associated with $24(67 \%)$ of all episodes of bacteremia, despite the fact that only $9 \%$ of all dialysis in the unit is conducted through this type of access. Table 2 calculates bacteremia rate by mode of dialysis and a risk of bacteremia per dialysis run. The bacteremia rate was 0.22 per patient dialysis year overall $(0.08$ for vascular graft dialysis patients and 1.56 for central venous catheter patients).

Of the 24 patients developing bacteremia while being dialyzed through a central line, the access site was in the subclavian position in 23 and the femoral site in one. Patients were undergoing central line dialysis because: no graft had yet been established (14 patients); a graft was established but not mature (nine patients); and presence of superficial graft site infection (one patient).

There was apparent clustering of infection suggestive of epidemic spread in the months of May and June 1988 (Figure 1), with $10(27 \%)$ of the bacteremic episodes occurring during these last two months of the study. However, a case control study of staff members failed to reveal any staff member as having a significant association with dialysis on/off procedures in bacteremic cases. 


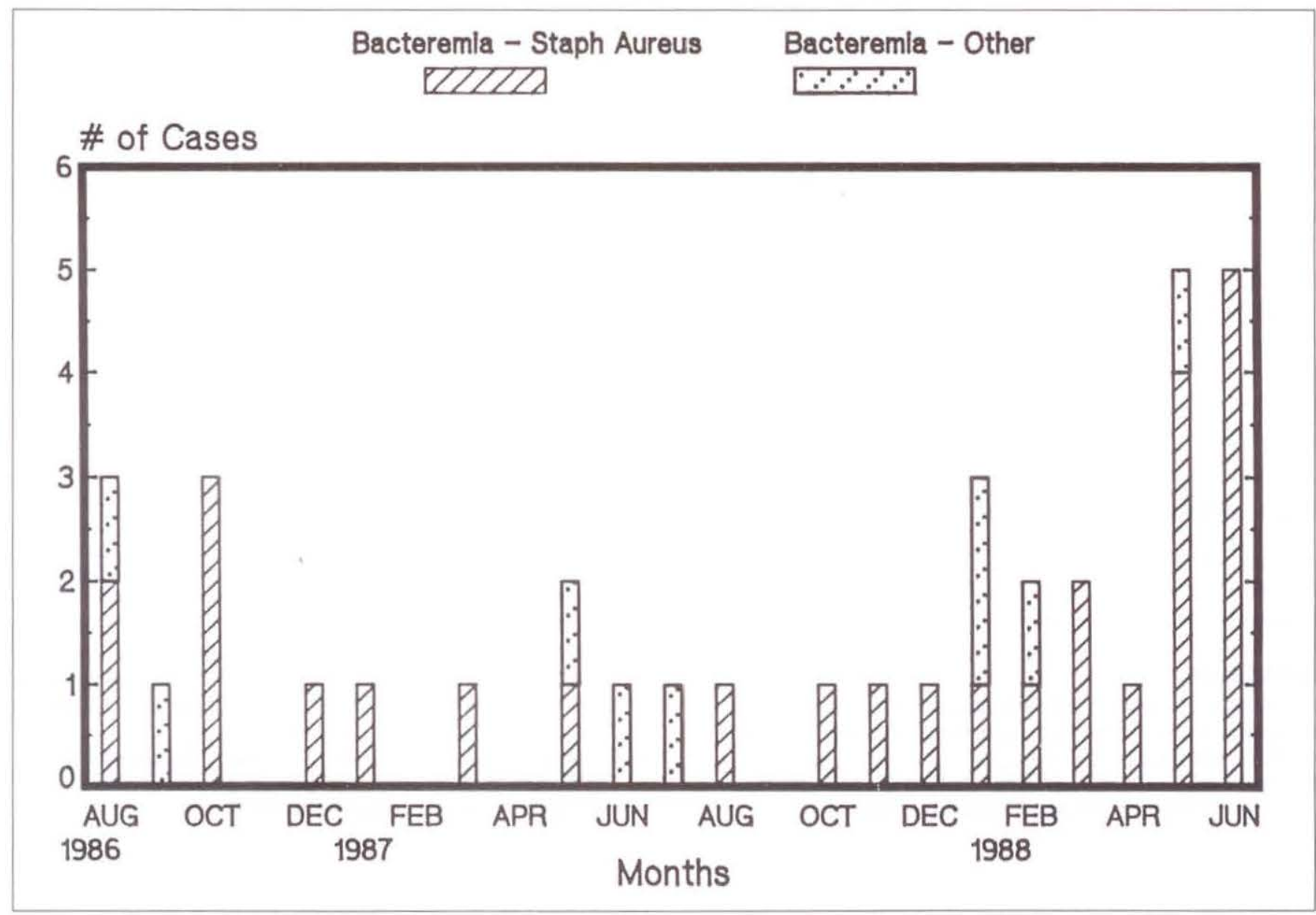

Figure 1) Bacteremias in hemodialysis patients from August 1986 to June 1988 at the University of Alberta Hospitals dialysis centre

Likewise, phage typing of the 10 isolates in this two month period failed to reveal a common isolate.

\section{DISCUSSION}

This study is similar to others documenting the extent of risk of bacteremia in hemodialysis patients (1,4). It is also similar in documenting the type of microorganisms causing these infections (1,4-6). Staph aureus accounted for $75 \%$ of all of the bacteremias seen in the 23 months of the study. Fortunately, the mortality rate related to these was quite low - only one death related to infection was seen. This is quite likely related to the close supervision patients undergoing incentre hemodialysis receive, probably resulting in prompt diagnosis and treatment of infection.

The major finding of this study was documentation of the greatly increased risk of bacteremia in patients undergoing hemodialysis via a central venous catheter. Since this was not a controlled study, it is possible that other factors associated with these patients are more important than the mode of dialysis itself. It seems unlikely that these possible other factors could completely account for this greatly increased risk. It is left to be concluded that central venous access dialysis should be avoided if at all possible, eg. by close following of patients with moderate degrees of renal impairment and creation of vascular grafts well in advance of possible commencement of hemodialysis. The vast majority of the centralline-related bacteremias (23 of 24) occurred in patients who either did not have a vascular graft in place or who had a graft not sufficiently mature to be used. Further steps should be taken to reduce the risk of dialysis via central catheters. As the access cannula is left in place for weeks in some cases, it is not difficult to conceptualize the process by which microorganisms might traverse the 'catheter wound' to enter the bloodstream. Other studies have demonstrated that colonization of skin at the insertion site of central venous catheters is a major source of organisms causing bacteremia (7). Staph aureus carriage on the skin has been demonstrated in 6 to $42 \%$ of the hemodialysis patient population $(8,9)$. Staph aureus remained on the skin after skin prepara- 
tion and has been found to be present in significantly heavier growth in persons with poor hygiene. Eliminating chronic Staph aureus carriage, either with intermittent cycles of oral rifampin and intranasal bacitracin (8) or more recently with intermittent or continuous intranasal mupirocin $(10,11)$, has been suggested to be useful in reducing the incidence of bacteremia due to this organism.

Body site selected for venous access has been shown to be an important variable. Femoral access sites should be avoided. The authors avoid femoral access if possible, and as a consequence

ACKNOWLEDGEMENTS: The authors thank the staff of the in-centre hemodialysis unit, University of Alberta Hospitals for their cooperation and for allowing observation of their patients.

\section{REFERENCES}

1. Dobkin JF, Miller MH, Steigbigel NH. Septicemia in patients on chronic hemodialysis. Ann Intern Med 1978:88:28-33,

2. Degoulet P. Legrain M, Reach I, et al. Mortality risk factors in patients treated by chronic hemodialysis. Nephron 1982:31:103-10.

3. Schwarzheek A, Brittinger WD. Henning GE. Slrauch M. Cannulation of subclavian vein for hemodialysis using Seldinger's technique. Trans Am Soc Artif Intern Organs 1978; 14:27-9.

4. Foissac-Gegoux P. Dumant A. Septicémies au cours du traitement par hémodialyse périodique. Nouv Presse Med 1974:3:151. (Lett)

5. Quarles LD, Rutsky EA, Rostand SG. Staphylococcus bacteremia in patients on chronic hemodialysis. Am J Kidney Dis 1985:6:412-9.

6. Meyrier A, Chevet D. Marsac J. Leroux-Rober C. Sraer J, Scetbon V. Staphylococcèmies chez les malades hêmodialysées. Nouv Presse Med 1973:2:2379-83.

7. Maki DG. Sources of infection with central venous catheters in an ICU: A prospective study. Program and Abstracts of the 28th Interscience Conference saw only one bacteremia in a patient dialyzing through this site. Increased frequency of manipulation of the catheter junction sites - for example, when the catheter is used for the administration of drugs, fluids or blood products - is also felt to be a risk factor and should likewise be avoided (12). Finally, the type of dressing applied to the cannula has recently been shown to be an important factor in increasing the risk of infection. Transparent semiocclusive dressings have been shown to increase the multiplication of bacteria under the dressing and lead to an increased risk of catheter infection (13).

on Antimicrobial Agents and Chemotherapy. Los Angeles, California. October 1988:157.

8. Yu VL, Goetz A, Wagener M, et al. Staphylococcus aureus nasal carriage and infection in patients on hemodialysis. N Engl J Med 1986:315:91-6.

9. Haplowitz L, Comstook J, Landwehr D, Dalton H, Mayhall C. Prospective study of microbial colonization of the nose and skin and infection of the vascular access site in hemodialysis patients. J Clin Microbiol 1989;26:1257-62.

10. Boelaert JR, DeBaere Y, Godard C. Van Landuyt HW. Eradication of Staphylococcus aureus in dialysis by nasal mupirocin. Program and Abstracts of the 29th Interscience Conference on Antimicrobial Agents and Chemotherapy, Houston. Texas. September 1989:315.

11. Holton D, Nicolle LE. Diley D. Bernstein K. Efficacy of mupirocin nasal ointment in eradicating Staphylococcus aureus in chronic hemodialysis patients. Program and Abstracts of the 29th Interscience Conference on Antimicrobial Agents and Chemotherapy. Houston. Texas. September 1989:315.

12. Maki DG. Sepsis arising from extrinsic contamination of the infusion and measures for control: In: Philips I, ed. Microbiological Hazards of Infusion Therapy. Lancaster: MTP Press, 1977:99-141.

13. Conly J, Grieve K, Peters B. A prospective randomized study comparing transparent and dry gauze dressings for central venous catheters. $\mathrm{J}$ Infect Dis 1989;159:310-9. 


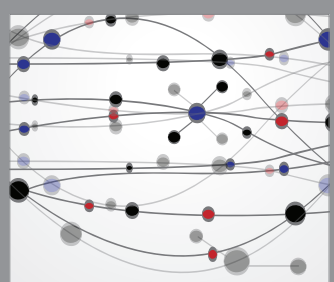

The Scientific World Journal
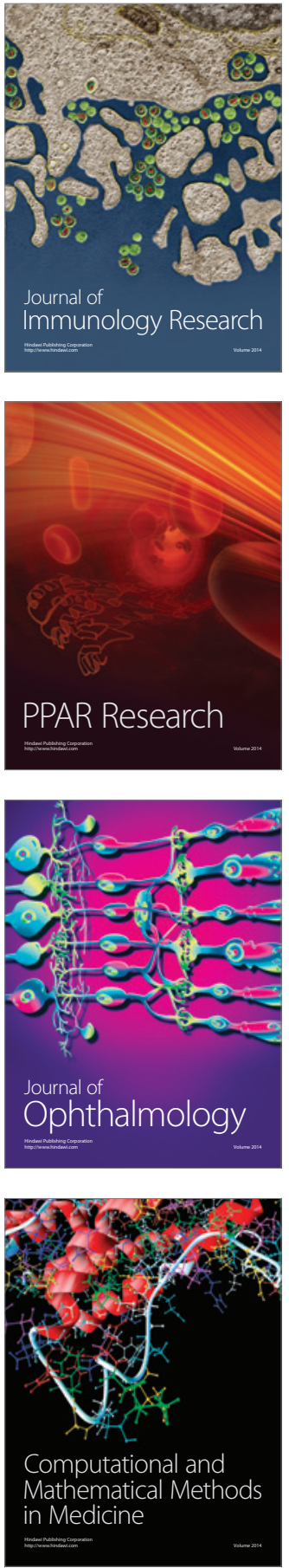

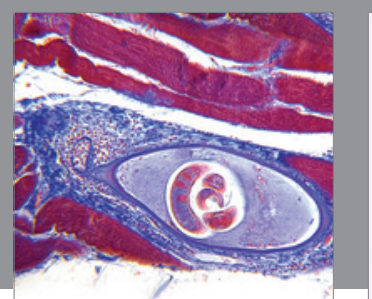

Gastroenterology Research and Practice

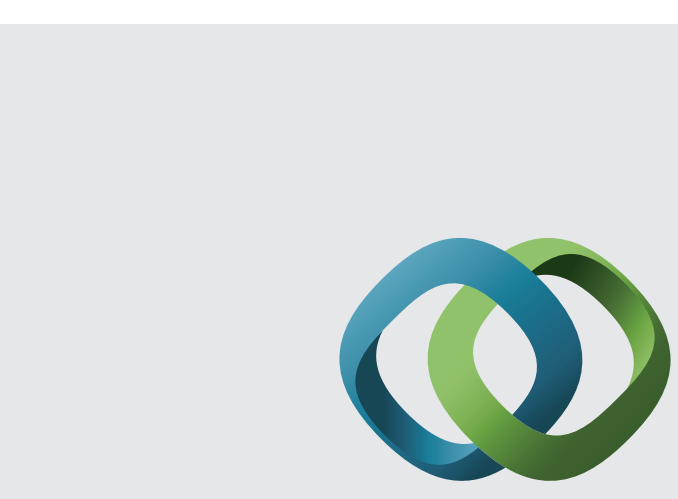

\section{Hindawi}

Submit your manuscripts at

http://www.hindawi.com
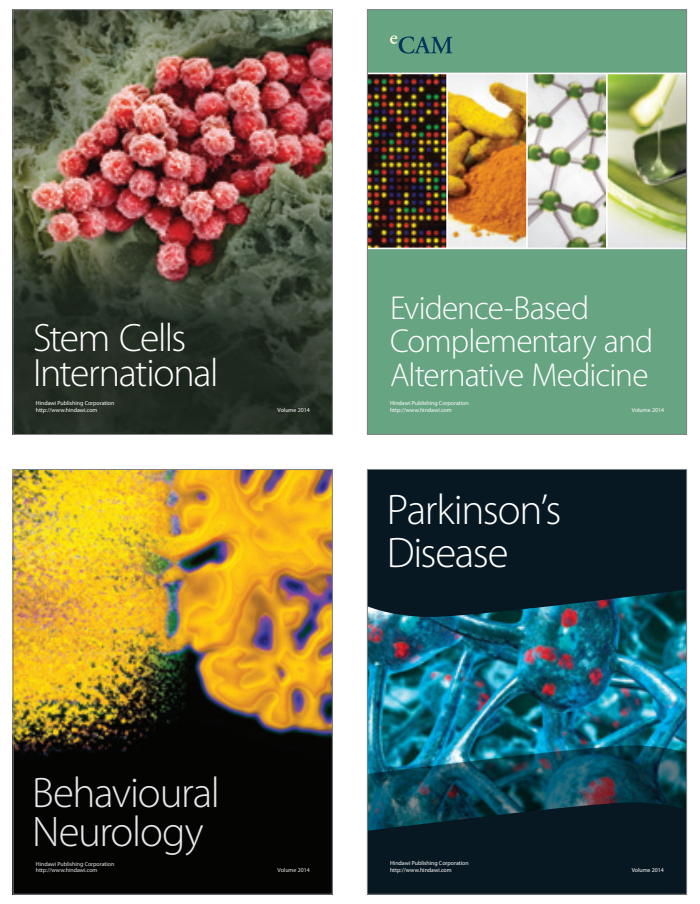
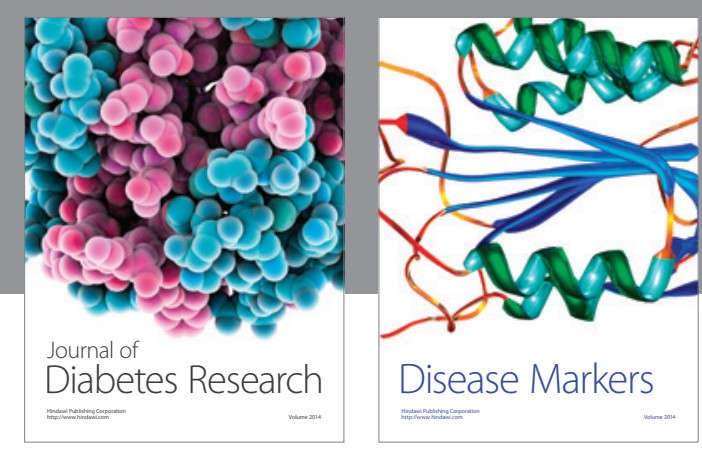

Disease Markers
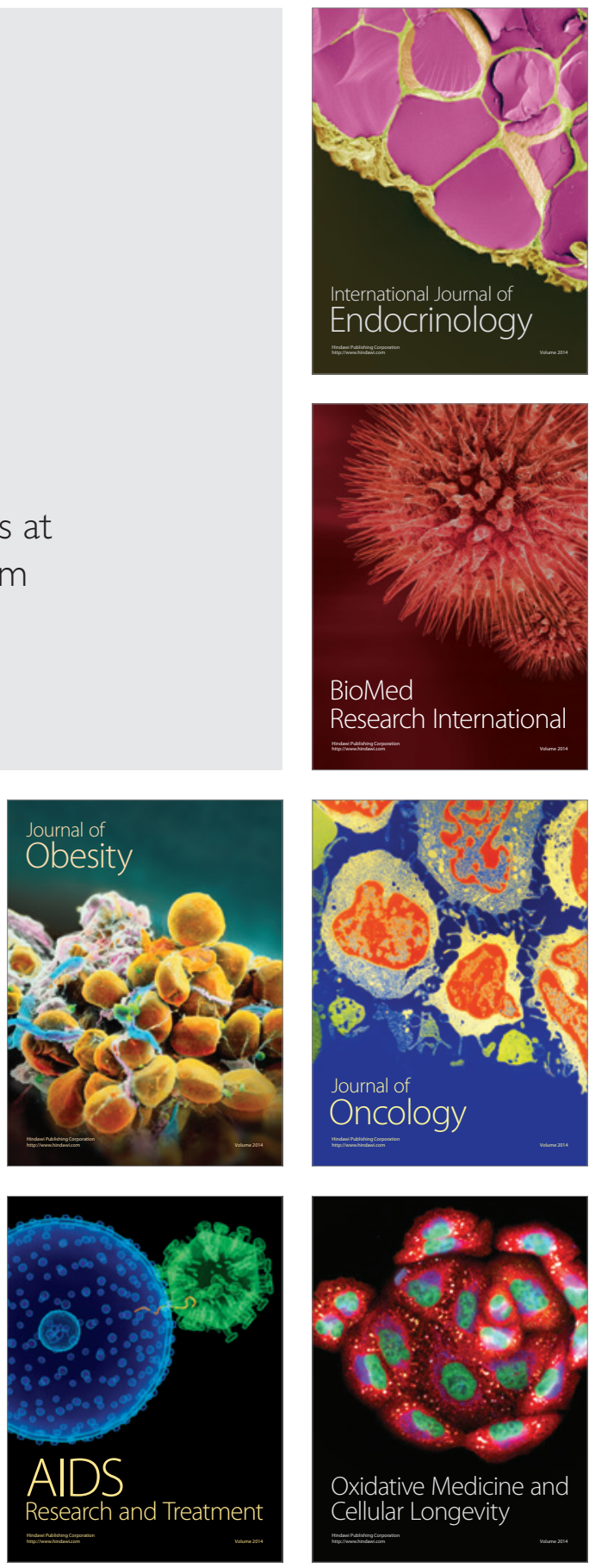